\title{
OPEN Leukocyte related parameters in older adults with metabolically healthy and unhealthy overweight or obesity
}

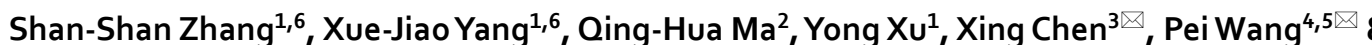
Chen-Wei Pan ${ }^{1 \bowtie}$

It remains unclear whether leukocyte-related parameters could be used as biomarkers to differentiate metabolically unhealthy overweight/obesity (MUO) from metabolically healthy overweight/obesity (MHO). We aimed to examine the differences in the distribution of leukocyte-related parameters between older adults with MHO and MUO and the correlations of leukocyte-related parameters with individual components of metabolic abnormality. In the Weitang Geriatric Diseases Study on older Chinese adults aged 60 years or above, 404 individuals with $\mathrm{MHO}$ and 480 with MUO contributed to the analysis. Overweight/obesity was defined as body mass index (BMI) of $25 \mathrm{~kg} / \mathrm{m}^{2}$ or more. MHO and MUO were discriminated based on the Adult Treatment Panel III (ATP III) criteria. Leukocyterelated parameters were assessed using an automated hematology analyzer. All leukocyte-related parameters except monocytes were elevated in MUO group compared with MHO group (all $P<0.05$ ). The prevalence of MUO increased by $24 \%$ with each $10^{9} / \mathrm{L}$ increase of leukocytes after adjusting for confounders in the multiple-adjusted model $(P<0.01)$ and each unit elevation of other parameters except lymphocytes and monocytes were significantly associated with the presence of MUO (all $P<0.01$ ). Trend tests revealed a linear trend for the association between MUO and all the leukocyterelated parameters (all $P$ for trend $<0.05$ ). Significant interactions between leukocyte-related parameters and sex on the presence of MUO were observed (all $P$ value for interaction $<0.05$ ). Higher leukocyte-related parameters were found in patients with MUO than those with MHO and were associated with higher prevalence of MUO which seems to be sex-dependent. Further studies are needed to see whether these parameters could be used as biomarkers for the screening or diagnosis for MUO in clinical or public health practice.

Overweight/obesity has been recognized as a major public health concern mainly affected by behavioral risk factors such as physical inactivity and dietary factors ${ }^{1,2}$. There are two subtypes of overweight/obesity, namely "metabolically healthy overweight/obesity" (MHO) and "metabolically unhealthy overweight/obesity" (MUO), which are differentiated by the absence or presence of metabolic disturbance ${ }^{1,3}$. The mortality observed with MHO was estimated to decrease by $30-50 \%$ compared to that with $\mathrm{MUO}^{4}$. Thus, figuring out cost-effective and easy-to-access biomarkers which could differentiate MUO from MHO, especially in older adults who were more vulnerable to MUO-related complications, is essential for health management of overweight/obesity in the communities.

Systemic low-grade and long-term inflammation has been suggested to play an important role in the pathological process of overweight/obesity. A study suggested that although some morbid obese were metabolically healthy, they also presented an altered rheological profile which is related with inflammation ${ }^{5}$. Previous studies have indicated that the concomitant inflammation activity was lower in MHO patients in contrast to those

\footnotetext{
${ }^{1}$ School of Public Health, Medical College of Soochow University, 199 Ren Ai Road, Suzhou 215123, China. ${ }^{2}$ The 3rd People's Hospital of Xiangcheng District, Suzhou 215134, China. ${ }^{3}$ Department of Children Health Care, Affiliated Suzhou Hospital of Nanjing Medical University, No.26, Dao Qian Road, Suzhou 215000, China. ${ }^{4}$ Department of Health Economics, School of Public Health, Fudan University, 130 Dong An Road, Shanghai 200032, China. ${ }^{5}$ Key Lab of Health Technology Assessment, National Health Commission of the People's Republic of China (Fudan University), Shanghai 200032, China. ${ }^{6}$ These authors contributed equally: Shan-Shan Zhang and Xue-Jiao Yang.『email: cx1708@126.com; wang_p@fudan.edu.cn; pcwonly@gmail.com
} 
with $\mathrm{MUO}^{1,6-8}$. For example, a proteomics study observed pro-inflammatory levels were decreased and levels of anti-inflammatory biomarkers were increased in individuals with $\mathrm{MHO}^{9}$. Karelis et al. ${ }^{10}$ observed a lower favorable inflammation state in MHO subjects, as attested by low CRP levels, suggesting that lower inflammation could play a role in the protective profile of the MHO individuals, and this may be associated to a lower risk for cardiovascular disease. Phillips et al. ${ }^{11}$ found reduced inflammatory status increased the likelihood of metabolic health, particularly among obese subjects according to a cross-sectional study conducted on 2047 men and women aged 45-74.

However, previous studies assessing the role of chronic inflammation in MUO mainly focused on biomarkers like $\mathrm{C}$ reactive protein $(\mathrm{CRP})^{3,6,9}$. CRP is widely known as an acute-phase response protein and thought as a bystander marker of inflammation, which did not play a direct role in the inflammatory process ${ }^{12}$. In addition, CRP is not a commonly measured biomarker in medical check-up programs among older adults, which limited its use from a public health perspective. Leukocyte-related parameters such as counts of total leukocytes, lymphocytes, monocytes, and neutrophils is another group of chronic inflammation biomarkers which are easy to obtain in medical check-up programs. There are limited studies comparing the distributions of leukocyterelated parameters between MHO and MUO groups among older adults. The lack of evidence highlights the pressing need to identify the specific leukocyte-related parameters which could differentiate the two subtypes of overweight/obesity, especially among older adults, among whom the harmful effect of chronic inflammation on overweight/obesity might be exaggerated ${ }^{13}$.

In this study, we examined the differences in the distribution of leukocyte-related parameters between older adults with MHO and MUO and the correlations of these parameters with each component of metabolic abnormality in a community-based study on older Chinese adults. These findings might have important implications for weight management and prevention of obesity-related cardiometabolic features among older populations.

\section{Materials and methods}

Study sample. This analysis is part of the Weitang Geriatric Diseases Study, which is a community-based study consisting of adults aged 60 years or older in the Weitang town of Suzhou, located in Eastern China. Detailed study protocol of the Weitang Geriatric Diseases Study has been described elsewhere ${ }^{14-16}$. In brief, each adult aged 60 years or older was invited to participate in the study based on local official records, while one was excluded if he or she was immigrant resident, lived there for less than six months or died. Of the 5613 eligible adults enrolled in the study, 4611 adults participated in the clinical examinations from Aug 2014 to Feb 2015 and 4579 participants with completed information contributed to the data collection ultimately. Among the 4579 participants, 884 individuals were defined to have overweight/obesity, which was defined as body mass index (BMI) of $25 \mathrm{~kg} / \mathrm{m}^{2}$ or more based on the WHO criteria ${ }^{17}$.

This study was conducted following tenets of the Helsinki Declaration and the approval was obtained from the Institutional Review Board of Soochow University. Written informed consent was obtained from all the participants before data collection.

Measurements of variables. Height of participants was measured in centimeters without shoes using a wall-mounted measurement tape and weight was observed in kilograms with light clothing through a digital scale. Blood pressure (BP) was measured at least 3 times after a rest interval of 5 min or longer by an automatic blood pressure monitor (Dinamap model Pro Series DP110X-RW, 100V2; GE Medical Systems Information Technologies, Inc., Milwaukee, Wisconsin, United States) and the average of the last two readings was calculated as the value of BP. Venous blood samples were collected after 12-h fasting for laboratory evaluations. The leukocyte-related parameters described as $\times 109$ cells/L was obtained using an automated hematology analyzer (XT-4000i, SYSMEX, Japan) and levels of HDL-C, TG, and fasting plasma glucose were measured with a chemistry analyzer (Roche cobas c 501). Characteristics of social-demographic were collected by trained staff via a pre-designed questionnaire such as age, sex, education level and marital status. Variables of lifestyle such as smoking, alcohol drinking and tea consumption were self-reported.

Definitions of MHO and MUO. Metabolically healthy and unhealthy overweight/obesity were discriminated using the Adult Treatment Panel III (ATP III) criteria ${ }^{18}$, in which the definition of MHO required individuals with overweight/obesity to have one or none of the following components: (1) systolic blood pressure (SBP) no lower than $130 \mathrm{mmHg}$ or diastolic blood pressure (DBP) no lower than $85 \mathrm{mmHg}$ or using antihypertensive drugs; (2) serum triglycerides (TG) of $1.7 \mathrm{mmol} / \mathrm{L}(150 \mathrm{mg} / \mathrm{dL})$ or more or use of lipid-lower drugs; (3) blood high-density lipoprotein cholesterol (HDL-C) less than $1.04 \mathrm{mmol} / \mathrm{L}(40 \mathrm{mg} / \mathrm{dL}$ ) in men and $1.29 \mathrm{mmol} / \mathrm{L}$ $(50 \mathrm{mg} / \mathrm{dL})$ in women; (4) fasting plasma glucose (FPG) of $7.0 \mathrm{mmol} / \mathrm{L}(126 \mathrm{mg} / \mathrm{dL})$ or higher or previously diagnosed diabetes mellitus or use of medications for diabetes. If the participant with overweight/obesity had two or more of the above components, he or she was considered to have MUO.

Statistical analysis. Continuous variables were described as mean \pm standard deviation (SD) while categorical ones were presented as numbers and percentages. The differences of leukocyte-related parameters between MHO and MUO were examined using the Student's t test (if normal distributed) or Mann-Whitney U test (if not normal distributed) after Shapiro and Wilk test conducted for estimation of normality distribution. Trend tests were used to examine the possible dose-response relationships of the proportion of MUO with levels of leukocyte-related parameters. Multivariable logistic regression models were fitted to estimate the associations between leukocyte-related parameters and the presence of MUO. Adjusted odds ratios (ORs) of higher quartiles compared with the lowest quartile for each unit increment of leukocyte-related parameters and their corresponding $95 \%$ confidence intervals (CIs) were calculated and presented. Only age and sex were adjusted in 


\begin{tabular}{|c|c|c|c|c|}
\hline & \multicolumn{3}{|c|}{ Overweight/obesity } & \multirow[b]{3}{*}{$P$ value ${ }^{*}$} \\
\hline & \multirow{2}{*}{\begin{tabular}{|l|} 
Participants \\
$(\mathbf{n}=\mathbf{8 8 4})$ \\
\end{tabular}} & \multirow{2}{*}{\begin{tabular}{|l|} 
Metabolic normality \\
$(n=404)$
\end{tabular}} & \multirow{2}{*}{\begin{tabular}{|l|} 
Metabolic abnormality \\
$(n=480)$
\end{tabular}} & \\
\hline & & & & \\
\hline Sex (women), \% & 47.40 & 39.10 & 54.40 & $<0.01$ \\
\hline Age, mean(SD), years & $66.39(5.29)$ & $66.62(5.46)$ & $66.20(5.14)$ & 0.34 \\
\hline $\mathrm{BMI}$, mean $(\mathrm{SD}), \mathrm{kg} / \mathrm{m}^{2}$ & $27.37(8.03)$ & $27.48(11.64)$ & $27.28(2.20)$ & 0.71 \\
\hline SBP, mean(SD), mmHg & $148.56(18.57)$ & $146.02(19.53)$ & $150.70(17.45)$ & $<0.01$ \\
\hline DBP, mean(SD), mmHg & $89.22(10.89)$ & $88.26(11.43)$ & $90.03(10.35)$ & $<0.05$ \\
\hline Fasting glucose, mean(SD), mmol/L & $5.88(1.42)$ & $5.47(0.69)$ & $6.22(1.45)$ & $<0.01$ \\
\hline Triglycerides, mean(SD), mmol/L & $1.63(0.93)$ & $1.13(0.34)$ & $2.05(1.05)$ & $<0.01$ \\
\hline HDL-cholesterol, mean(SD), mmol/L & $1.3(0.34)$ & $1.49(0.32)$ & $1.14(0.27)$ & $<0.01$ \\
\hline Current smoking, $\%$ & 26.70 & 28.50 & 25.20 & 0.11 \\
\hline Alcohol consumption, \% & 27.10 & 32.90 & 22.30 & $<0.01$ \\
\hline Tea consumption, $\%$ & 41.00 & 45.80 & 36.90 & $<0.01$ \\
\hline Hypertension, \% & 66.20 & 54.70 & 75.80 & $<0.01$ \\
\hline Diabetes mellitus, \% & 12.00 & 0.70 & 21.50 & $<0.01$ \\
\hline No formal education, $\%$ & 44.50 & 44.60 & 44.40 & 0.92 \\
\hline
\end{tabular}

Table 1. Demographic, somatometric, and laboratory parameters in MHO and MUO individuals. SD standard deviation, $B M I$ body mass index, $S B P$ systolic blood pressure, $D B P$ diastolic blood pressure, $H D L$ high-density lipoprotein. ${ }^{*} P$ values represent difference in characteristics by metabolic syndrome status based on analysis of t-test.

model 1. We additionally adjusted for potential confounders such as education and lifestyle variables (smoking, alcohol intake and tea consumption). The associations between leukocyte-related parameters and individual metabolic components in both MHO and MUO groups were investigated using a partial correlation analysis. A likelihood ratio test was performed to investigate the potential interaction effects between leukocyte-related parameters and sex on MUO and sex-stratified analysis was further performed if the interaction effect was statistically significant.

A two-sided $P$ value of less than 0.05 was considered as a statistically significant level for all analyses. The procedure of statistical analysis was conducted using the Statistics Analysis System (version 9.4; Cary, NC) and SPSS version 21.0 (SPSS Inc., Chicago, IL, USA).

\section{Results}

Among the 884 individuals with overweight/obesity, 404 (45.70\%) were defined as MHO and the other 480 (54.30\%) were defined as MUO. In general, MUO group had significantly higher SBPs (146.02 $\mathrm{mmHg}$ vs. $150.70 \mathrm{mmHg} ; P<0.01)$, DBPs $(88.26 \mathrm{mmHg}$ vs. $90.03 \mathrm{mmHg} ; P<0.05)$, FPGs $(5.47 \mathrm{mmol} / \mathrm{L}$ vs. $6.22 \mathrm{mmol} / \mathrm{L}$; $P<0.01)$ and lower HDL-Cs $(1.14 \mathrm{mmol} / \mathrm{L}$ vs. $1.49 \mathrm{mmol} / \mathrm{L} ; P<0.01)$ as compared with MHO groups. (Table 1$)$.

The distributions of leukocyte-related parameters by metabolic status as defined by the ATP III criteria are displayed in Table 2. In all participants with overweight/obesity, all leukocyte-related parameters except monocytes were elevated in MUO group compared with MHO group (all $P<0.05$ ). Sex-stratified analysis revealed that the pattern of associations between leukocyte-related parameters and MHO/MUO was different between men and women. The leukocyte count, neutrophil count and basophil count were significantly higher in MUO group than that in MHO group in men (all $P<0.05$ ), while women with MUO were more likely to have increased count of neutrophils and eosinophils and decreased count of leukocytes (all $P<0.05$ ) compared with their counterparts with MHO.

Table 3 shows the multivariate-adjusted associations of leukocyte-related parameters with the presence of MUO in logistic regression models. The prevalence of MUO increased by $24 \%$ with each $10^{9} / \mathrm{L}$ increase of leukocytes after adjusting for confounders in the multiple-adjusted model $(P<0.01)$. Besides, each unit elevation of other parameters except lymphocytes and monocytes were significantly associated with the presence of MUO (all $P<0.01$ ). All ORs of higher quartiles compared with the lowest quartile for each unit increment of monocytes and eosinophils were significantly above 1.00 (all $P<0.05$ ). Trend tests revealed that there was a linear trend for the association between MUO and all the leukocyte-related parameters (all $P$ for trend $<0.05$ ).

A partial correlation analysis revealed significant and positive correlations between neutrophil count and $\operatorname{SBP}(r=0.10)$ and FBG $(r=0.11)$ in subjects with MUO (Table 4$)$. Neutrophil count was positively correlated with SBP, but not with DBP. Positive correlations were found between TG and counts of leukocytes, neutrophils, eosinophils and basophils. Eosinophil count was negatively correlated with HDL-C $(r=-0.10)$.

We further examined the relationship between leukocyte-related parameters and the presence MUO in men and women. Modest positive associations between leukocyte-related parameters except basophils and the presence of MUO were observed in both men and women. Furthermore, significant interactions between leukocyterelated parameters and sex on MUO were observed (all $P$ value for interaction <0.05; Fig. 1). Eosinophil count significantly elevated in MUO group in women $(\mathrm{OR}=29.94,95 \% \mathrm{CI}[2.70,332.26])$ but not in men $(\mathrm{OR}=2.93$, $95 \%$ CI $[0.79,10.97]$, Fig. 1). On the contrary, basophil count was found to be significantly associated with a 


\begin{tabular}{|c|c|c|c|c|}
\hline & \multicolumn{3}{|c|}{ Overweight/obesity } & \multirow[b]{2}{*}{$P$ value ${ }^{\star}$} \\
\hline & Participants & Metabolic normality & Metabolic abnormality & \\
\hline All persons & $\mathrm{n}=884$ & $\mathrm{n}=404$ & $\mathrm{n}=480$ & \\
\hline Leukocyte, mean (SD), $\times 10^{9} / \mathrm{L}$ & $5.62(1.44)$ & $5.43(1.54)$ & $5.79(1.33)$ & $<0.01$ \\
\hline Lymphocyte, mean (SD), $\times 10^{9} / \mathrm{L}$ & $1.76(0.77)$ & $1.70(0.96)$ & $1.81(0.56)$ & $<0.05$ \\
\hline Monocyte, mean (SD), $\times 10^{9} / \mathrm{L}$ & $0.33(0.13)$ & $0.33(0.12)$ & $0.33(0.14)$ & 0.62 \\
\hline Neutrophil, mean $(\mathrm{SD}), \times 10^{9} / \mathrm{L}$ & $3.36(1.04)$ & $3.24(1.03)$ & $3.46(1.04)$ & $<0.01$ \\
\hline Eosinophil, mean $(\mathrm{SD}), \times 10^{9} / \mathrm{L}$ & $0.14(0.13)$ & $0.13(0.13)$ & $0.15(0.13)$ & $<0.05$ \\
\hline Basophil, mean $(\mathrm{SD}), \times 10^{9} / \mathrm{L}$ & $0.03(0.02)$ & $0.03(0.02)$ & $0.03(0.02)$ & $<0.05$ \\
\hline Men & $\mathrm{n}=465$ & $\mathrm{n}=246$ & $\mathrm{n}=219$ & \\
\hline Leukocyte, mean (SD), $\times 10^{9} / \mathrm{L}$ & $5.68(1.54)$ & $5.49(1.66)$ & $5.90(1.37)$ & $<0.01$ \\
\hline Lymphocyte, mean (SD), $\times 10^{9} / \mathrm{L}$ & $1.82(0.92)$ & $1.66(1.14)$ & $1.79(0.56)$ & 0.13 \\
\hline Monocyte, mean $(\mathrm{SD}), \times 10^{9} / \mathrm{L}$ & $0.35(0.15)$ & $0.35(0.12)$ & $0.36(0.17)$ & 0.42 \\
\hline Neutrophil, mean (SD), $\times 10^{9} / \mathrm{L}$ & $3.42(1.05)$ & $3.30(1.02)$ & $3.55(1.08)$ & $<0.05$ \\
\hline Eosinophil, mean $(\mathrm{SD}), \times 10^{9} / \mathrm{L}$ & $0.15(0.15)$ & $0.14(0.15)$ & $0.17(0.15)$ & 0.08 \\
\hline Basophil, mean $(\mathrm{SD}), \times 10^{9} / \mathrm{L}$ & $0.03(0.02)$ & $0.03(0.02)$ & $0.04(0.02)$ & $<0.01$ \\
\hline Women & $\mathrm{n}=419$ & $\mathrm{n}=158$ & $\mathrm{n}=261$ & \\
\hline Leukocyte, mean $(\mathrm{SD}), \times 10^{9} / \mathrm{L}$ & $5.57(1.32)$ & $5.43(1.54)$ & $5.79(1.33)$ & $<0.01$ \\
\hline Lymphocyte, mean (SD), $\times 10^{9} / \mathrm{L}$ & $1.81(0.56)$ & $1.76(0.57)$ & $1.83(0.56)$ & 0.20 \\
\hline Monocyte, mean (SD), $\times 10^{9} / \mathrm{L}$ & $0.30(0.10)$ & $0.29(0.10)$ & $0.31(0.09)$ & 0.15 \\
\hline Neutrophil, mean $(\mathrm{SD}), \times 10^{9} / \mathrm{L}$ & $3.30(1.03)$ & $3.14(1.04)$ & $3.40(1.01)$ & $<0.05$ \\
\hline Eosinophil, mean $(\mathrm{SD}), \times 10^{9} / \mathrm{L}$ & $0.13(0.10)$ & $0.11(0.09)$ & $0.14(0.11)$ & $<0.01$ \\
\hline Basophil, mean $(\mathrm{SD}), \times 10^{9} / \mathrm{L}$ & $0.03(0.02)$ & $0.03(0.02)$ & $0.03(0.02)$ & 0.33 \\
\hline
\end{tabular}

Table 2. Leukocyte-related parameters of study participants by metabolic status. ${ }^{\star} P$ values represent difference in characteristics by metabolic syndrome status based on analysis of t-test.

higher prevalence of MUO in men $(\mathrm{OR}=1.02,95 \% \mathrm{CI}[1.01,1.03])$ whereas no significant association was observed in women $(\mathrm{OR}=1.01,95 \% \mathrm{CI}[0.99,1.02]$; Fig. 1$)$.

\section{Discussion}

In this study, we observed that most of the leukocyte-related parameters were elevated in MUO group compared with $\mathrm{MHO}$ group, indicating that high levels of inflammation might be involved in MUO pathology. Moreover, the pattern of leukocyte-MHO/MUO associations might be different in men and women. Our study suggested that elevated leukocyte-related parameters might be related to a higher likelihood of MUO which seems to be sex-dependent.

Added to the above results, age and BMI were not significantly different between MHO and MUO group. There may be three possible reasons. Firstly, the subjects were all overweight or obese people over 60 years old, in which case there might be a correlation between age and metabolic status, but it was not inevitable, so did the relationship between BMI and metabolic status. Moreover, all participants were selected from the same town and thus they were likely to have certain homogeneity. Finally, the absence of such differences may also be due to the limitation of sample size.

Some previous studies have reported clinically significant differences in leukocyte-related parameters between MHO and MUO individuals and the findings seems to be inconsistent ${ }^{6,8,19,20}$. Our study showed a significant elevated level of leukocyte counts in the MUO group compared with their metabolically healthy counterparts, which was consistent with part of previous literatures ${ }^{8,11,21}$. Some other studies reported non-significant findings. For instance, Lynch et al. ${ }^{20}$ did not detect any differences in leukocyte count, but reported significantly higher levels of natural killer and cytotoxic T lymphocytes in MHO subjects which may protect against malignancy, infection and metabolic diseases. Wang et al. ${ }^{22}$ observed no significant differences in the counts of lymphocytes and neutrophils between MHO and MUO. A Mendelian Randomization study suggested the associations of blood eosinophil count and metabolic diseases may not be causal ${ }^{23}$. Moreover, contrary to our study, absolute counts of monocytes were higher in MUO subjects compared with MHO subjects as Christou et al. ${ }^{5}$ found in their study, while in their another study the difference appeared non-significant consistent with our results ${ }^{24}$. These conflicting findings may be explained by differences in methodological issues such as disparities in characteristics of the participants, inflammatory profiling and definitions of $\mathrm{MHO} / \mathrm{MUO}^{6}$. Our study added novel evidence in this area by observing a dose-response relationship between leukocyte-related parameters such as leukocytes, neutrophils, eosinophils and basophils and the presence of MUO, which was not reported by previous studies.

The mechanism underlying the observations in the current study remains to be elucidated. Old adults tends to have elevated levels of free radicals and reactive oxidative species (ROS) and were more likely to be harmed by oxidative stress ${ }^{25}$. Leukocytes could promote oxidative stress and inflammation ${ }^{26}$ and have been demonstrated to play a role in insulin resistance (IR) $)^{1,27}$. IR is widely considered as the etiology of obesity and metabolic abnormality and may lead to higher gathering of inflammatory markers such as total leukocyte ${ }^{28,29}$. Moreover, shorter 


\begin{tabular}{|c|c|c|c|c|}
\hline & \multicolumn{2}{|c|}{ Age-and sex-adjusted } & \multicolumn{2}{|l|}{ Multiple adjusted $^{*}$} \\
\hline & OR $(95 \% \mathrm{CI})$ & $P$ value & OR $(95 \% \mathrm{CI})$ & $P$ value \\
\hline Leukocyte, $10^{9} / \mathrm{L}$ & $1.24(1.12,1.38)$ & $<0.01$ & $1.24(1.12,1.38)$ & $<0.01$ \\
\hline $\mathrm{Q} 1(\leq 4.72)$ & Ref & & Ref & \\
\hline \begin{tabular}{|l|} 
Q2(4.73-5.42) \\
\end{tabular} & $1.46(1.00,2.14)$ & 0.05 & $1.42(0.97,2.09)$ & 0.07 \\
\hline Q3(5.43-6.35) & $1.89(1.29,2.78)$ & $<0.01$ & $1.85(1.25,2.73)$ & $<0.01$ \\
\hline $\mathrm{Q} 4(>6.35)$ & $2.45(1.66,3.63)$ & $<0.01$ & $2.48(1.66,3.68)$ & $<0.01$ \\
\hline P for trend & \multicolumn{2}{|l|}{$<0.01$} & \multicolumn{2}{|l|}{$<0.01$} \\
\hline Lymphocyte, $10^{9} / \mathrm{L}$ & $1.26(0.99,1.59)$ & 0.06 & $1.25(0.98,1.59)$ & 0.08 \\
\hline $\mathrm{Q} 1(\leq 1.37)$ & Ref & & Ref & \\
\hline \begin{tabular}{|l|} 
Q2(1.38-1.67) \\
\end{tabular} & $1.44(0.99,2.10)$ & 0.06 & $1.44(0.98,2.11)$ & 0.06 \\
\hline \begin{tabular}{|l|} 
Q3(1.68-2.06) \\
\end{tabular} & $1.76(1.20,2.57)$ & $<0.01$ & $1.83(1.24,2.70)$ & $<0.01$ \\
\hline $\mathrm{Q} 4(>2.06)$ & $2.01(1.36,2.96)$ & $<0.01$ & $2.04(1.38,3.03)$ & $<0.01$ \\
\hline P for trend & \multicolumn{2}{|l|}{$<0.01$} & \multicolumn{2}{|l|}{$<0.01$} \\
\hline Monocyte, $10^{9} / \mathrm{L}$ & $2.47(0.78,7.79)$ & 0.12 & $2.83(0.90,8.89)$ & 0.08 \\
\hline $\mathrm{Q} 1(\leq 0.25)$ & Ref & & Ref & \\
\hline Q2(0.26-0.31) & $1.77(1.22,2.59)$ & $<0.01$ & $1.72(1.17,2.52)$ & $<0.01$ \\
\hline Q3(0.32-0.37) & $1.51(1.03,2.23)$ & $<0.05$ & $1.53(1.04,2.26)$ & $<0.05$ \\
\hline $\mathrm{Q} 4(>0.37)$ & $1.53(1.04,2.25)$ & $<0.05$ & $1.56(1.06,2.30)$ & $<0.05$ \\
\hline P for trend & \multicolumn{2}{|l|}{0.06} & \multicolumn{2}{|l|}{$<0.05$} \\
\hline Neutrophil, $10^{9} / \mathrm{L}$ & $1.28(1.12,1.46)$ & $<0.01$ & $1.28(1.12,1.47)$ & $<0.01$ \\
\hline $\mathrm{Q} 1(\leq 2.63)$ & Ref & & Ref & \\
\hline Q2(2.64-3.20) & $1.45(0.99,2.13)$ & 0.06 & $1.41(0.96,2.08)$ & 0.08 \\
\hline \begin{tabular}{|l|} 
Q3(3.21-3.87) \\
\end{tabular} & $1.56(1.07,2.29)$ & $<0.05$ & $1.51(1.02,2.22)$ & $<0.05$ \\
\hline Q4(>3.87) & $2.21(1.49,3.27)$ & $<0.01$ & $2.22(1.49,3.30)$ & $<0.05$ \\
\hline $\mathrm{P}$ for trend & \multicolumn{2}{|l|}{$<0.01$} & \multicolumn{2}{|l|}{$<0.01$} \\
\hline Eosinophil, $10^{9} / \mathrm{L}$ & $6.16(1.89,20.08)$ & $<0.01$ & $6.40(1.94,21.17)$ & $<0.01$ \\
\hline $\mathrm{Q} 1(\leq 0.07)$ & Ref & & Ref & \\
\hline Q2(0.08-0.11) & $1.70(1.18,2.46)$ & $<0.01$ & $1.62(1.12,2.35)$ & $<0.05$ \\
\hline \begin{tabular}{|l|} 
Q3(0.12-0.18) \\
\end{tabular} & $1.64(1.13,2.40)$ & $<0.05$ & $1.64(1.12,2.41)$ & $<0.05$ \\
\hline $\mathrm{Q} 4(>0.18)$ & $1.98(1.36,2.90)$ & $<0.01$ & $2.01(1.37,2.96)$ & $<0.01$ \\
\hline P for trend & \multicolumn{2}{|l|}{$<0.01$} & \multicolumn{2}{|l|}{$<0.01$} \\
\hline Basophil, $10^{6} / \mathrm{L}$ & $1.01(1.00,1.02)$ & $<0.01$ & $1.01(1.00,1.02)$ & $<0.01$ \\
\hline Q1( $(\leq 20)$ & Ref & & Ref & \\
\hline Q2(21-30) & $1.21(0.85,1.71)$ & 0.29 & $1.17(0.83,1.67)$ & 0.37 \\
\hline Q3(31-40) & $1.33(0.91,1.96)$ & 0.15 & $1.29(0.87,1.91)$ & 0.21 \\
\hline Q4(>40) & $1.58(1.09,2.30)$ & $<0.05$ & $1.64(1.12,2.40)$ & $<0.05$ \\
\hline P for trend & \multicolumn{2}{|l|}{$<0.05$} & \multicolumn{2}{|l|}{$<0.01$} \\
\hline
\end{tabular}

Table 3. Adjusted Relationships of Leukocyte-related Parameters to the Prevalence of MUO. *Adjusted for age, sex, smoking, tea consumption, alcohol consumption, and education level.

leukocyte telomere length (LTL) has been reported to be related to increased IR and oxidative stress ${ }^{30}$. Lubrano et al. ${ }^{31}$ reported a possible transition mechanism of MUO from MHO involved leukocyte-related parameters. Granulocytes and monocytes are primed by oxidized low density lipoprotein ${ }^{32}$, and then produce large amount of ROS and pro-inflammatory cytokines like IL-6 and TNF- $\alpha$. Activated leukocytes in MHO individuals secrete excessive cytokines, thus prompting leukocyte migration into target tissues or organs for the initiation and maintenance of metabolic abnormality such as adipose tissue, pancreas and liver. Lipid oxidation may also induce adipogenesis and fat production by adipocyte ${ }^{26}$, creating vicious pathological loop of increased fat production and accumulation and metabolic disturbance mediated by leukocyte-related parameters. Additionally, leukocytes such as basophils were found to express receptors for leptin, a kind of adipokines secreted by adipocytes which affect energy expenditure, glucose and lipid metabolism and as well plays an important role in the pathophysiology of metabolic syndrome ${ }^{33,34}$.

The present study also found that the effects of leukocyte-related parameters on MUO significantly differed between men and women. First, a significant higher level of leukocyte count in MUO individuals was observed in men, while in women the count of leukocytes in MHO individuals was significantly higher than that of MUO individuals. Second, higher eosinophil count was significantly associated with higher prevalence of MUO in women but not in men, whereas basophil count was only found to be significantly associated with a higher prevalence of MUO in men. Gender difference in metabolic syndrome was also found but not discussed by 


\begin{tabular}{|c|c|c|c|c|c|c|}
\hline & BMI & SBP & DBP & Triglyceride & HDL-cholesterol & Glucose \\
\hline \multicolumn{7}{|l|}{ MHO } \\
\hline Leukocyte, mean $(\mathrm{SD}), \times 10^{9} / \mathrm{L}$ & -0.05 & 0.08 & 0.03 & $0.14^{*}$ & -0.05 & 0.02 \\
\hline Lymphocyte, mean (SD), $\times 10^{9} / \mathrm{L}$ & -0.01 & 0.01 & 0.01 & 0.01 & 0.02 & 0.07 \\
\hline Monocyte, mean $(\mathrm{SD}), \times 10^{9} / \mathrm{L}$ & -0.01 & -0.03 & 0.00 & 0.10 & -0.05 & -0.10 \\
\hline Neutrophil, mean $(\mathrm{SD}), \times 10^{9} / \mathrm{L}$ & -0.06 & $0.11^{*}$ & 0.04 & $0.17^{*}$ & -0.07 & -0.04 \\
\hline Eosinophil, mean $(\mathrm{SD}), \times 10^{9} / \mathrm{L}$ & -0.04 & -0.09 & -0.03 & $0.13^{*}$ & $-0.10^{*}$ & 0.10 \\
\hline Basophil, mean $(\mathrm{SD}), \times 10^{6} / \mathrm{L}$ & -0.05 & 0.06 & 0.03 & $0.12^{*}$ & -0.02 & -0.04 \\
\hline \multicolumn{7}{|l|}{ MUO } \\
\hline Leukocyte, mean (SD), $\times 10^{9} / \mathrm{L}$ & 0.02 & 0.09 & -0.06 & 0.02 & 0.00 & $0.10^{*}$ \\
\hline Lymphocyte, mean (SD), $\times 10^{9} / \mathrm{L}$ & 0.03 & 0.02 & -0.04 & 0.07 & -0.01 & 0.03 \\
\hline Monocyte, mean $(\mathrm{SD}), \times 10^{9} / \mathrm{L}$ & 0.04 & -0.04 & -0.04 & 0.02 & -0.08 & 0.05 \\
\hline Neutrophil, mean $(\mathrm{SD}), \times 10^{9} / \mathrm{L}$ & 0.00 & $0.10^{*}$ & -0.04 & -0.02 & 0.01 & $0.11^{*}$ \\
\hline Eosinophil, mean $(\mathrm{SD}), \times 10^{9} / \mathrm{L}$ & 0.05 & 0.04 & -0.04 & 0.03 & -0.03 & -0.05 \\
\hline Basophil, mean $(\mathrm{SD}), \times 10^{6} / \mathrm{L}$ & -0.01 & 0.06 & -0.06 & 0.07 & 0.03 & -0.05 \\
\hline
\end{tabular}

Table 4. Correlations between metabolic risk factors and leukocyte-related parameters from MHO and MUO elders. Adjusted for age, sex, smoking, tea consumption, alcohol consumption, and education level. BMI body mass index, SBP systolic blood pressure, $D B P$ diastolic blood pressure, $H D L$ high-density lipoprotein. ${ }^{\star} P<0.05$.

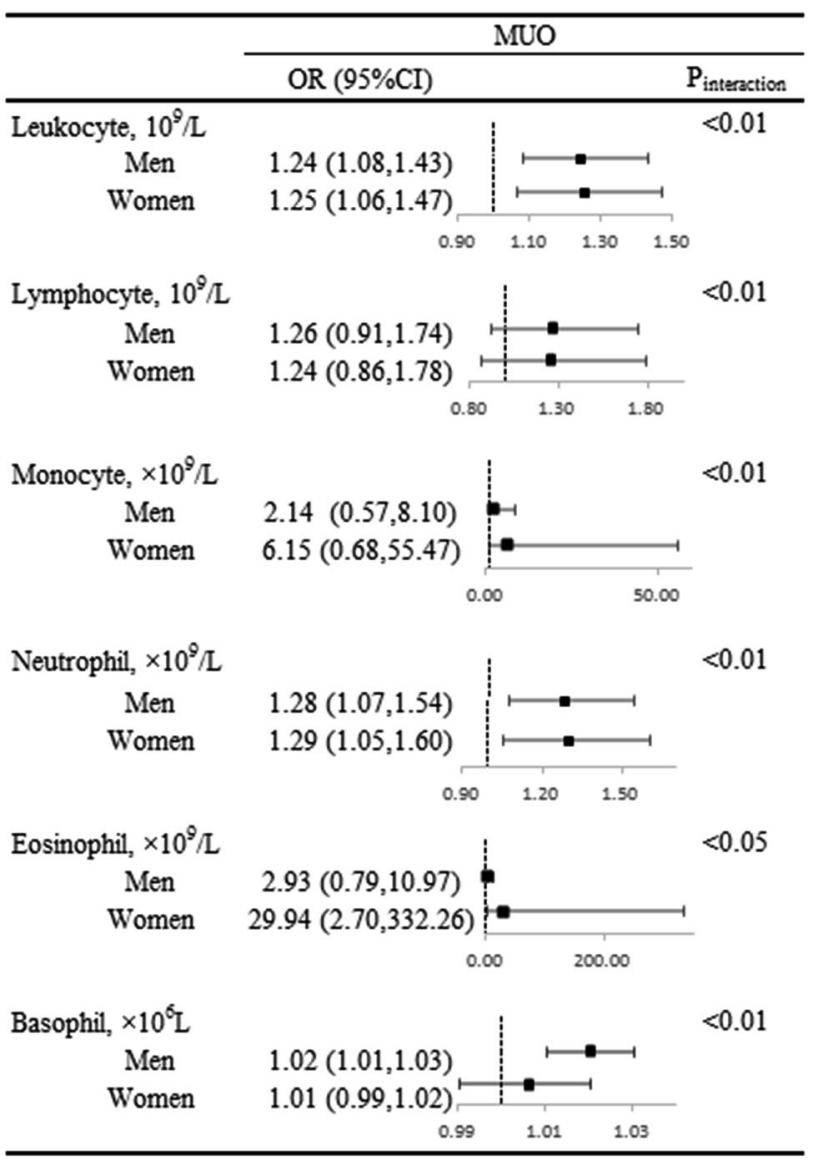

Figure 1. Subgroup analyses of the association between leukocyte-related parameters and MUO. Interactions between leukocyte-related parameters and sex on the MUO were tested by the likelihood ratio test with adjustment for the same variables as multiple models in Table 3 in addition to sex. Odds ratio and 95\% CIs were showed by forest plot. 
others $^{35}$. These inter-gender differences may be accounted for the differences in the behavioral and physiological variants between men and women. First, one reason for gender differences might be men had a higher frequency of smoking than women did in China, as cigarette smoking increases leukocyte count ${ }^{36}$. Second, the women subjects in our study were mostly postmenopausal. The body composition changes during menopausal transition including increased fat mass, leading to reduction in circulating adiponectin that is expressed in adipose tissue and correlated with many metabolic processes ${ }^{37}$. Premature telomere shortening have been discussed as potential mechanisms linking metabolic disorders, and postmenopausal women had decreased estrogen levels, which may prevent telomerase from being activated to catalyze the synthesis and extension of telomeres ${ }^{38}$. Particularly, shortened LTL might accelerate the leukocyte senescence and death and decrease leukocyte count. Third, the different variation of IL- 6 and leptin by adipose tissue inflammation between men and women might also contribute to such sex-related association. A recent study demonstrated a positive association of adipose tissue inflammation with leptin and IL-6 specifically in men and a negative association of adipose tissue inflammation with adiponectin in women ${ }^{39}$. Fourth, sex-specific differences in mitochondrial function and free radical homeostasis could also contribute to the etiology of $\mathrm{MUO}^{40}$. Further studies are warranted in this area.

Considering the growing burden of overweigh/obesity worldwide and the increased death risk of MUO versus $\mathrm{MHO}^{4}$, our findings may have both public health implications. From a public health perspective, there is an urgent need to identify cost-effective biomarkers for MUO screening among older adults in rural communities, where the socioeconomic status is low. Our findings suggested that leukocyte-related parameters, a group of common and easy-to-access blood biomarkers, might be used by physicians and public health practitioners for differentiating MUO patients from MHO. On the other hand, we have to acknowledge that the finding of the study was preliminary and far from conclusive. Whether leukocyte-related parameters could be used as biomarkers for the screening or diagnosis for MUO in clinical or public health practice needs further clarifications.

To our best knowledge, this study was the first which comprehensively examine the differences in the distribution of a group of leukocyte-related parameters between older adults with MHO and MUO in a large-size community-based sample. However, several limitations in this study need to be noted. First, the study was crosssectional and whether leukocyte-related parameters could predict the transition to from MHO to MUO could not be determined. Second, history of inflammation and infection of the participants were not collected, which may have altered the characteristics of inflammatory markers.

In conclusion, all leukocyte-related parameters except monocytes were significantly elevated in MUO individuals compared with MHO individuals and gender-specific patterns regarding the associations were observed. Further well-designed prospective studies are needed to assess the role of leukocyte-related parameters in the transition from MHO to MUO. The biological mechanisms underlying the observations should be elucidated as well.

Received: 2 July 2020; Accepted: 27 January 2021

Published online: 25 February 2021

\section{References}

1. Iacobini, C., Pugliese, G., Blasetti Fantauzzi, C., Federici, M. \& Menini, S. Metabolically healthy versus metabolically unhealthy obesity. Metabolism 92, 51-60. https://doi.org/10.1016/j.metabol.2018.11.009 (2019).

2. Jia, A. et al. Prevalence and cardiometabolic risks of normal weight obesity in Chinese population: A nationwide study. Nutr. Metab. Cardiovasc. Dis. 28, 1045-1053. https://doi.org/10.1016/j.numecd.2018.06.015 (2018).

3. Ctoi, A. F. et al. Metabolically healthy versus unhealthy morbidly obese: Chronic inflammation, nitro-oxidative stress, and insulin resistance. Nutrients https://doi.org/10.3390/nu10091199 (2018).

4. Ortega, F. B. et al. The intriguing metabolically healthy but obese phenotype: Cardiovascular prognosis and role of fitness. Eur. Heart J. 34, 389-397. https://doi.org/10.1093/eurheartj/ehs174 (2013).

5. Christou, K. A. et al. Metabolically healthy obesity is characterized by a proinflammatory phenotype of circulating monocyte subsets. Metab. Syndr. Relat. Disord. 17, 259-265. https://doi.org/10.1089/met.2018.0132 (2019).

6. Phillips, C. M. Metabolically healthy obesity: Definitions, determinants and clinical implications. Rev. Endocr. Metab. Disord. 14, 219-227. https://doi.org/10.1007/s11154-013-9252-x (2013).

7. Kouvari, M. et al. Transition from metabolically benign to metabolically unhealthy obesity and 10-year cardiovascular disease incidence: The ATTICA cohort study. Metabolism 93, 18-24. https://doi.org/10.1016/j.metabol.2019.01.003 (2019).

8. Vaya, A. et al. Metabolic alterations in morbid obesity. Influence on the haemorheological profile. Clin. Hemorheol. Microcirc. 48, 247-255. https://doi.org/10.3233/CH-2011-1417 (2011).

9. Doumatey, A. P. et al. Proinflammatory and lipid biomarkers mediate metabolically healthy obesity: A proteomics study. Obes. (Silver Spring) 24, 1257-1265. https://doi.org/10.1002/oby.21482 (2016).

10. Karelis, A. D. et al. The metabolically healthy but obese individual presents a favorable inflammation profile. J. Clin. Endocrinol. Metab. 90, 4145-4150. https://doi.org/10.1210/jc.2005-0482 (2005).

11. Phillips, C. M. \& Perry, I. J. Does inflammation determine metabolic health status in obese and nonobese adults?. J. Clin. Endocrinol. Metab. 98, E1610-1619. https://doi.org/10.1210/jc.2013-2038 (2013).

12. Ilhan, N., Ilhan, N., Ilhan, Y., Akbulut, H. \& Kucuksu, M. C-reactive protein, procalcitonin, interleukin-6, vascular endothelial growth factor and oxidative metabolites in diagnosis of infection and staging in patients with gastric cancer. World J. Gastroenterol. 10, 1115-1120. https://doi.org/10.3748/wjg.v10.i8.1115 (2004).

13. Alam, I., Ng, T. P. \& Larbi, A. Does inflammation determine whether obesity is metabolically healthy or unhealthy? The aging perspective. Mediators Inflamm. 2012, 456456. https://doi.org/10.1155/2012/456456 (2012).

14. Pan, C. W. et al. Evaluating health-related quality of life impact of chronic conditions among older adults from a rural town in Suzhou China. Arch. Gerontol. Geriatr. 76, 6-11. https://doi.org/10.1016/j.archger.2018.01.008 (2018).

15. Gu, Y. J. et al. Tea consumption is associated with cognitive impairment in older Chinese adults. Aging Ment. Health 22, 1232-1238. https://doi.org/10.1080/13607863.2017.1339779 (2018).

16. Pan, C. W. et al. Cognitive dysfunction and health-related quality of life among older Chinese. Sci. Rep. 5, 17301. https://doi. org/10.1038/srep17301 (2015).

17. Obesity: preventing and managing the global epidemic. Report of a WHO consultation. World Health Organ. Tech. Rep. Ser. 894, $1-253(2000)$. 
18. Expert Panel on Detection, E. \& Treatment of High Blood Cholesterol in, A. Executive summary of the third report of the national cholesterol education program (NCEP) expert panel on detection, evaluation, and treatment of high blood cholesterol in adults (adult treatment panel III). JAMA 285, 2486-2497, https://doi.org/10.1001/jama.285.19.2486 (2001).

19. Wildman, R. P. et al. Body size phenotypes and inflammation in the Women's health initiative observational study. Obesity (Silver Spring) 19, 1482-1491. https://doi.org/10.1038/oby.2010.332 (2011).

20. Lynch, L. A. et al. Are natural killer cells protecting the metabolically healthy obese patient?. Obesity (Silver Spring) 17, 601-605. https://doi.org/10.1038/oby.2008.565 (2009).

21. Baeg, M. K., Ko, S. H., Ko, S. Y., Jung, H. S. \& Choi, M. G. Obesity increases the risk of erosive esophagitis but metabolic unhealthiness alone does not: A large-scale cross-sectional study. BMC Gastroenterol. 18, 82. https://doi.org/10.1186/s12876-018-0814-y (2018).

22. Wang, X. L. et al. Peripheral invariant natural killer T cell deficiency in metabolically unhealthy but normal weight versus metabolically healthy but obese individuals. J. Int. Med. Res. 44, 1272-1282. https://doi.org/10.1177/0300060516663778 (2016).

23. Amini, M. et al. Blood eosinophil count and metabolic, cardiac and pulmonary outcomes: A mendelian randomization study. Twin Res. Hum. Genet. 21, 89-100. https://doi.org/10.1017/thg.2018.6 (2018).

24. Christou, K. A. et al. The regulation of serum resistin levels in metabolically healthy and unhealthy obese individuals. Hormones (Athens) 19, 523-529. https://doi.org/10.1007/s42000-020-00201-1 (2020).

25. Boengler, K., Kosiol, M., Mayr, M., Schulz, R. \& Rohrbach, S. Mitochondria and ageing: Role in heart, skeletal muscle and adipose tissue. J. Cachexia Sarcopenia Muscle 8, 349-369. https://doi.org/10.1002/jcsm.12178 (2017).

26. Mazor, R. et al. Primed polymorphonuclear leukocytes constitute a possible link between inflammation and oxidative stress in hyperlipidemic patients. Atherosclerosis 197, 937-943. https://doi.org/10.1016/j.atherosclerosis.2007.08.014 (2008).

27. Nakanishi, N., Suzuki, K. \& Tatara, K. White blood cell count and clustered features of metabolic syndrome in Japanese male office workers. Occup. Med. (Lond.) 52, 213-218. https://doi.org/10.1093/occmed/52.4.213 (2002).

28. Razani, B., Chakravarthy, M. V. \& Semenkovich, C. F. Insulin resistance and atherosclerosis. Endocrinol. Metab. Clin. N. Am. 37, 603-621. https://doi.org/10.1016/j.ecl.2008.05.001 (2008)

29. Yang, X. J. et al. Leukocyte-related parameters in older adults with metabolic syndrome. Endocrine https://doi.org/10.1007/s1202 0-020-02243-2 (2020).

30. Demissie, S. et al. Insulin resistance, oxidative stress, hypertension, and leukocyte telomere length in men from the Framingham Heart Study. Aging Cell 5, 325-330. https://doi.org/10.1111/j.1474-9726.2006.00224.x (2006).

31. Lubrano, C. et al. Integrated haematological profiles of redox status, lipid, and inflammatory protein biomarkers in benign obesity and unhealthy obesity with metabolic syndrome. Oxid. Med. Cell Longev. 2015, 490613. https://doi.org/10.1155/2015/490613 (2015).

32. Trpkovic, A. et al. Oxidized low-density lipoprotein as a biomarker of cardiovascular diseases. Crit. Rev. Clin. Lab Sci. 52, 70-85. https://doi.org/10.3109/10408363.2014.992063 (2015).

33. Suzukawa, M. et al. Leptin enhances survival and induces migration, degranulation, and cytokine synthesis of human basophils. J. Immunol. 186, 5254-5260. https://doi.org/10.4049/jimmunol.1004054 (2011).

34. Ghadge, A. A. \& Khaire, A. A. Leptin as a predictive marker for metabolic syndrome. Cytokine 121, 154735. https://doi. org/10.1016/j.cyto.2019.154735 (2019).

35. Monserrat-Mesquida, M. et al. Metabolic syndrome is associated with oxidative stress and proinflammatory state. Antioxidants (Basel) https://doi.org/10.3390/antiox9030236 (2020).

36. Ishizaka, N., Ishizaka, Y., Toda, E., Nagai, R. \& Yamakado, M. Association between cigarette smoking, white blood cell count, and metabolic syndrome as defined by the Japanese criteria. Intern. Med. 46, 1167-1170. https://doi.org/10.2169/internalmedicin e.46.0136 (2007)

37. Vichinsartvichai, P. \& Sirirat, S. Hematologic parameters as the predictors for metabolic syndrome in perimenopausal and postmenopausal women living in urban area: A preliminary report. Prz Menopauzalny 15, 90-95. https://doi.org/10.5114/pm.2016.61191 (2016).

38. Mangge, H. et al. Subcutaneous adipose tissue distribution and telomere length. Clin. Chem. Lab Med. 57, 1358-1363. https://doi. org/10.1515/cclm-2018-0801 (2019).

39. Ter Horst, R. et al. Sex-specific regulation of inflammation and metabolic syndrome in obesity. Arterioscler. Thromb. Vasc. Biol. 40, 1787-1800. https://doi.org/10.1161/ATVBAHA.120.314508 (2020).

40. Khalifa, A. R. et al. Sex-specific differences in mitochondria biogenesis, morphology, respiratory function, and ROS homeostasis in young mouse heart and brain. Physiol. Rep. https://doi.org/10.14814/phy2.13125 (2017).

\section{Acknowledgements}

This study was supported by the Science and Technology Bureau of Xiangcheng District in Suzhou, China [Grant No. XJ201706] and the Health Commission of Suzhou [Grant No. GSWS2019090].

\section{Author contributions}

S.-S.Z. and X.-J.Y. conducted the statistical analyses and wrote manuscript. Q.-H.M. and Y.X. contributed to data collection and interpretation of the data. X.C., P.W. and C.-W.P. conceived and designed the study and revised manuscript. All authors read and approved the final manuscript.

\section{Competing interests}

The authors declare no competing interests.

\section{Additional information}

Correspondence and requests for materials should be addressed to X.C., P.W. or C.-W.P.

Reprints and permissions information is available at www.nature.com/reprints.

Publisher's note Springer Nature remains neutral with regard to jurisdictional claims in published maps and institutional affiliations. 
(c) (i) Open Access This article is licensed under a Creative Commons Attribution 4.0 International cc) License, which permits use, sharing, adaptation, distribution and reproduction in any medium or format, as long as you give appropriate credit to the original author(s) and the source, provide a link to the Creative Commons licence, and indicate if changes were made. The images or other third party material in this article are included in the article's Creative Commons licence, unless indicated otherwise in a credit line to the material. If material is not included in the article's Creative Commons licence and your intended use is not permitted by statutory regulation or exceeds the permitted use, you will need to obtain permission directly from the copyright holder. To view a copy of this licence, visit http://creativecommons.org/licenses/by/4.0/.

(C) The Author(s) 2021 